OPEN ACCESS

Edited by:

Miguel A. Barboza

University of Costa Rica, Costa Rica

Reviewed by:

Vignan Yogendrakumar University of Ottawa, Canada

Alexander Tsiskaridze

Tbilisi State University, Georgia

*Correspondence:

Lili Song

Isong@georgeinstitute.org.cn

Craig S. Anderson

canderson@georgeinstitute.org.au

tThese authors have contributed equally to this work

Specialty section:

This article was submitted to

Stroke,

a section of the journa

Frontiers in Neurology

Received: 30 March 2021

Accepted: 12 July 2021

Published: 27 August 2021

Citation:

Chen C, Song L, Yang J, Lindley R, Robinson T, Arima H, Chalmers J, Anderson CS and Wang X (2021)

Regional Differences in Early BP Management After Acute Ischemic

Stroke in the ENCHANTED

International Randomized Controlled

Trials. Front. Neurol. 12:687862.

doi: 10.3389/fneur.2021.687862

\section{Regional Differences in Early BP Management After Acute Ischemic Stroke in the ENCHANTED International Randomized Controlled Trials}

\author{
Chen Chen ${ }^{1,2,3}$, Lili Song ${ }^{1,2 *}$, Jie Yang ${ }^{4}$, Richard Lindley ${ }^{1,5}$, Thompson Robinson ${ }^{6}$, \\ Hisatomi Arima $^{7}$, John Chalmers ${ }^{1}$, Craig S. Anderson ${ }^{1,2,8 *+}$ and Xia Wang ${ }^{1}$ for the \\ ENCHANTED Investigators
}

${ }^{1}$ The George Institute for Global Health, Faculty of Medicine, University of New South Wales, Newtown, NSW, Australia, ${ }^{2}$ The George Institute China at Peking University Health Science Centre, Beijing, China, ${ }^{3}$ Department of Neurology, Shanghai East Hospital, School of Medicine, Tongji University, Shanghai, China, ${ }^{4}$ Department of Neurology, the First Affiliated Hospital of Chengdu Medical College, Chengdu, China, ${ }^{5}$ Sydney Medical School, University of Sydney, Sydney, NSW, Australia, ${ }^{6}$ Department of Cardiovascular Sciences and NIHR Biomedical Research Centre, University of Leicester, Leicester, United Kingdom, ${ }^{7}$ Department of Preventive Medicine and Public Health, Fukuoka University, Fukuoka, Japan, ${ }^{8}$ Department of Neurology, Royal Prince Alfred Hospital, Sydney, NSW, Australia

Background and Aims: Epidemiological studies show significant variations in hypertension management within and between countries. The level of regional variation in early blood pressure (BP) management after acute stroke is uncertain.

Methods: Data are from the Enhanced Control of Hypertension and Thrombolysis Stroke Study (ENCHANTED), a partial-factorial, international randomized controlled trial of thrombolysis-eligible acute ischemic stroke (AIS) patients with elevated systolic $\mathrm{BP}$ (SBP >150 $\mathrm{mmHg}$ ) assigned to intensive (target SBP 130-140 $\mathrm{mmHg}$ ) vs. guideline-recommended (SBP $<180 \mathrm{mmHg}$ ) treatment; BP management was compared among four regions: Western countries (Italy/United Kingdom/Spain/Australia), China (mainland), other Asia (Hong Kong/Taiwan/Singapore/Thailand/Vietnam/India), and South America (Chile/Brazil/Colombia).

Results: These analyses included 2,196 AIS [38\% women, mean age 67 (12) years] patients. Commonly used intravenous BP-lowering agents were labetalol, nitroglycerin, and topical nitrates in Western countries; urapidil and sodium nitroprusside in China; nicardipine in other Asian countries; and sodium nitroprusside and labetalol in South America. Chinese patients were less likely to receive BP-lowering treatment in the first $24 \mathrm{~h}$ and be treated with multiple agents although they had smaller magnitude of SBP reduction and lower SBP variability.

Conclusion: Regional variations in early BP management in acute stroke translated into differences in early BP control parameters.

Keywords: stroke, thrombolysis, blood pressure, regional difference, clinical trial 


\section{INTRODUCTION}

Elevated blood pressure (BP) is one of the leading modifiable risk factors for stroke, including both intracerebral hemorrhage (ICH) and acute ischemic stroke (AIS) (1). In acute stroke, $75 \%$ of patients have high $\mathrm{BP}$, and $50 \%$ of those have a prior history of hypertension $(2,3)$. Large-scale epidemiological studies demonstrate significant variations in hypertension management at the country level and that within countries $(4,5)$. However, evidence on the variation in early BP management after acute stroke is scarce.

In the context of thrombolysis and BP management in AIS, the Enhanced Control of Hypertension and Thrombolysis in Stroke Study (ENCHANTED) $(6,7)$ enrolled patients at 110 hospitals in 14 countries. Most Asian participants were from middle income countries. This trial provides an ideal population to understand the regional differences in early BP management after AIS world widely.

TABLE 1 | Baseline characteristics.

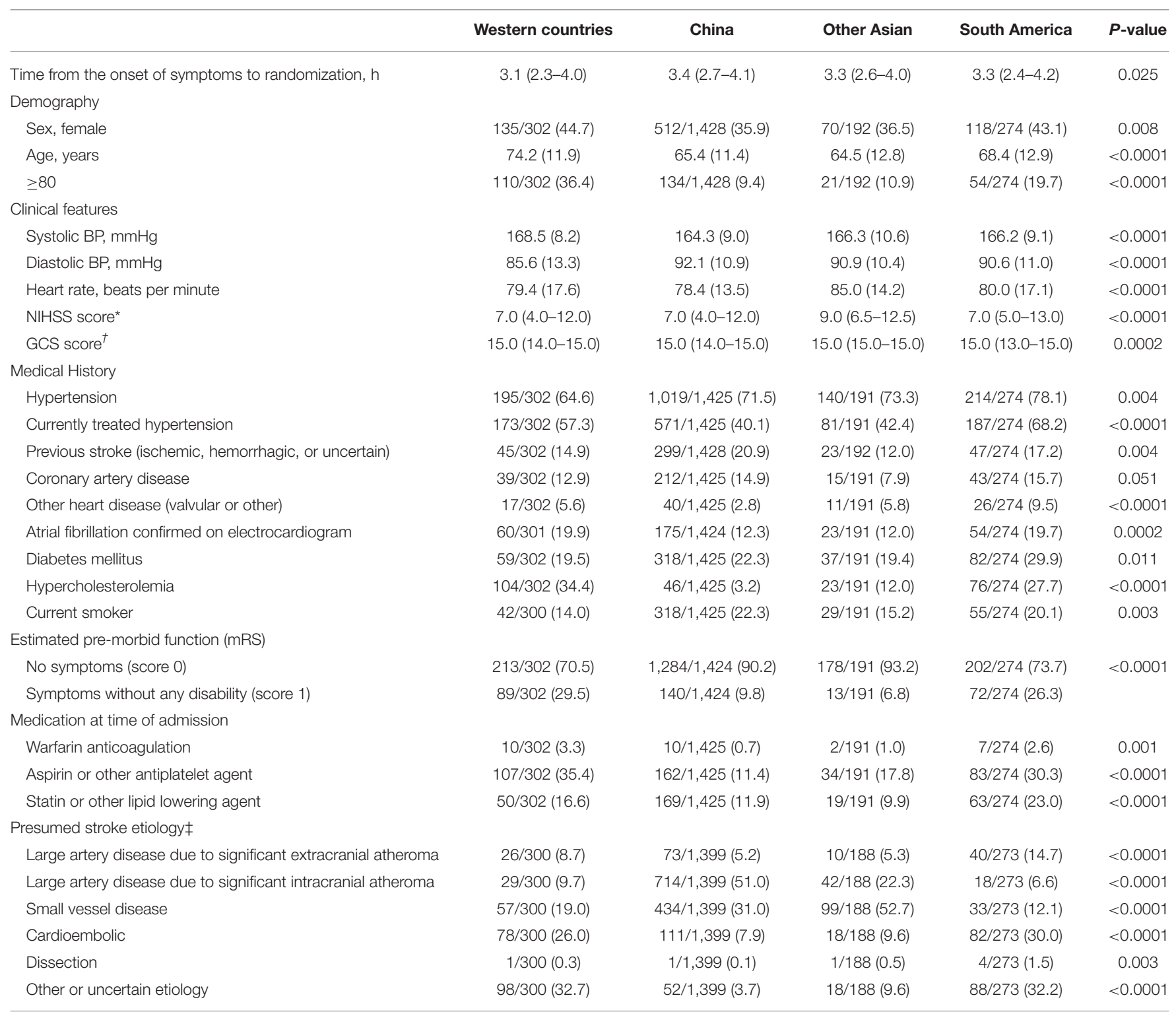

Data are $n(\%)$, mean (SD), or median (IQR). P-values are based on Chi-square, T-test, or Wilcoxon signed-rank test.

BP, blood pressure; CT, computerized tomography; GCS, Glasgow coma scale; MRI, magnetic resonance imaging; mRS, modified Rankin scale; NIHSS, National Institutes of Health Stroke Scale.

*Scores on the National Institutes of Health stroke scale (NIHSS) range from 0 to 42, with higher scores indicating more severe neurological deficit.

${ }^{\dagger}$ Scores on the Glasgow coma scale (GCS) range from 15 (normal) to 3 (deep coma).

$¥$ Diagnosis according to the clinician's interpretation of clinical features and results of investigations at the time of separation from hospital. 


\section{MATERIALS AND METHODS}

\section{Study Design}

ENCHANTED $(6,7)$ was an international, $2 \times 2$ partialfactorial, multicenter, prospective, randomized, open-label, blinded-endpoint (PROBE) trial. All ENCHANTED participants had a clinical diagnosis of AIS that was confirmed by brain imaging, were aged $\geq 18$ years, and fulfilled local criteria for thrombolysis treatment. In the BP arm, a total of 2,196 participants with systolic BP (SBP) $>150 \mathrm{mmHg}$ and time from onset within $6 \mathrm{~h}$ were randomly assigned to intensive (target SBP 130-140 mmHg) or guideline-recommended (SBP $<180$ mmHg) BP management between March 3, 2012, to April 30, 2018. In addition, the treating clinician had uncertainty over the benefits and risks of the intensity of $\mathrm{BP}$ control during and for up to $72 \mathrm{~h}$ (or hospital discharge or death if this occurred earlier) after thrombolytic treatment. A management strategy of BP-lowering treatment protocol in the ENCHANTED trial was based on locally available intravenous (IV, bolus and infusion), oral and topical medications (7). All patients were managed in an acute stroke unit or alternative environment with appropriate staffing and monitoring and received best practice management according to local guidelines. The use of endovascular thrombectomy, which increased in clinical practice during the course of the trial, was permitted.

\section{Measurements}

Countries were grouped into four regions: China (mainland), Western countries (United Kingdom, Italy, Norway, Australia), other Asia (South Korea, Hong Kong Area, Taiwan Area, Singapore, Thailand, Vietnam), and South America (Chile, Brazil, Colombia).
For each participant, summary measures of SBP control in the first $24 \mathrm{~h}$ were (i) "achieved SBP," the mean of SBP measures within the $24 \mathrm{~h}$ ("achieved" for short); (ii) "variability of SBP," the standard deviation (SD) of the measures within the $24 \mathrm{~h}$ ("variability" for short); (iii) "magnitude of absolute reduction of SBP," the difference between randomization SBP and the lowest attained SBP within the $24 \mathrm{~h}$ ("magnitude" for short).

\section{Data Analysis}

Generalized linear model (GLM) was used to investigate the difference in hemodynamic variables in the first $24 \mathrm{~h}$ between the four regions. The model was adjusted for age, sex, baseline SBP, baseline NIHSS score, comorbidities of prestroke disability, hypercholesterolemia, atrial fibrillation (AF), antithrombotic therapy, and randomized BP-lowering treatment. All statistical analyses were performed using SAS version 9.3 (SAS Institute, Cary, NC, USA).

\section{RESULTS}

A total of 2,196 ENCHANTED participants (38\% female) of mean age 67 years (SD 12.2) were included in these analyses. Median time from symptom onset to randomization was $3.3 \mathrm{~h}$ (IQR 2.6 to 4.1 ). Key baseline characteristics are provided in Table 1. There were 302, 1,428, 192, and 274 patients from Western countries, China, other Asia, and South America, respectively. Patients from Asia were younger and less female. Chinese patients had more previous stroke and stroke due to significant intracranial atheroma but were less likely to have history of AF and hypercholesterolemia, have strokes due to cardio embolism, and take antithrombotic agents.

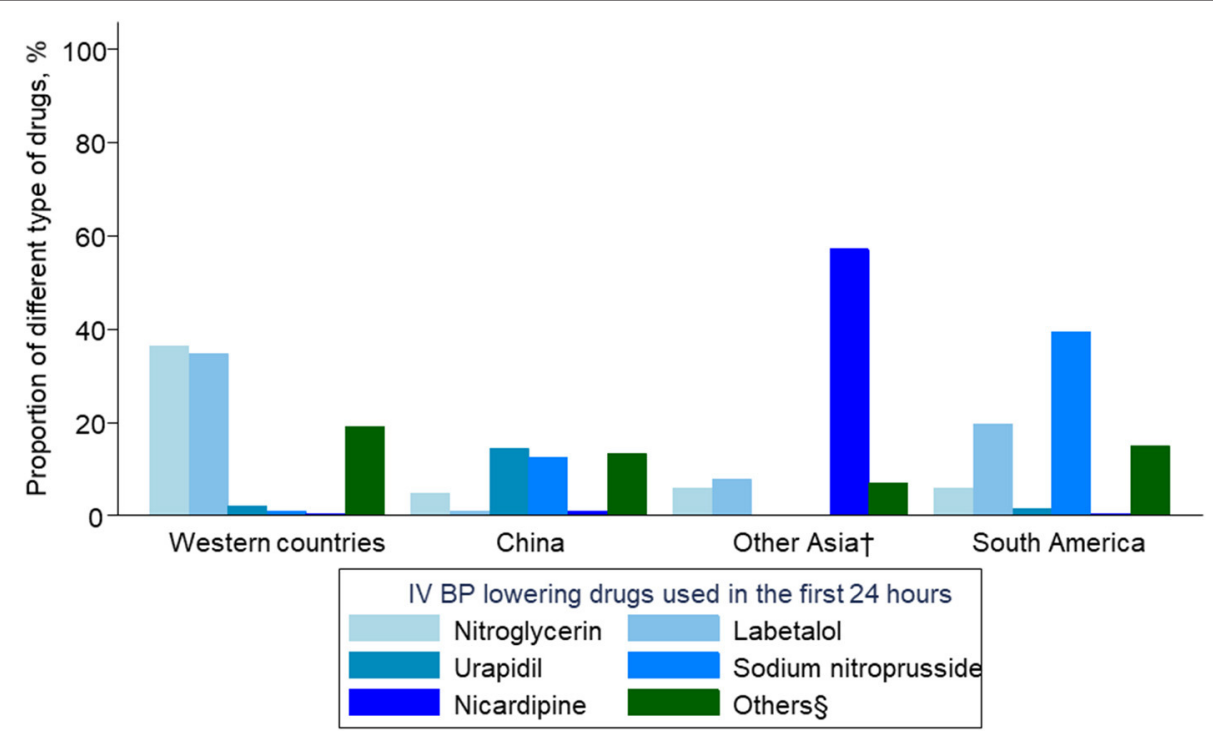

FIGURE 1 | Intravenous blood pressure-lowering drugs used in the first $24 \mathrm{~h}$. BP denotes blood pressure; IV intravenous. ${ }^{\dagger}$ South Korea/Hong Kong area/Taiwan area/Singapore/Thailand/Vietnam. §metoprolol, atenolol, clevidipine, nimodipine, nifedipine, urapidil, isodinitrate, frusemide, prazosin, hydralazine, clonidine, and enalapril. 
TABLE 2 | BP-lowering treatment by region in ENCHANTED.

\begin{tabular}{|c|c|c|c|c|c|}
\hline & Western countries & China & Other asian & South America & $P$-value \\
\hline $\mathrm{BP}$ lowering in the first $24 \mathrm{~h}$ after randomization & 241/302 (79.8) & $867 / 1,412(61.4)$ & $149 / 191(78.0)$ & $203 / 274(74.1)$ & $<0.0001$ \\
\hline Intravenous agent used, n(\%) & 176/302 (58.3) & $661 / 1,412(46.8)$ & 133/191 (69.6) & 190/274 (69.3) & $<0.0001$ \\
\hline Bolus on day 1 & 120/302 (39.7) & 243/1,411 (17.2) & 38/191 (19.9) & $72 / 272(26.5)$ & $<0.0001$ \\
\hline Infusion on day 1 & 102/302 (33.8) & 430/1,411 (30.5) & $124 / 191(64.9)$ & $142 / 273(52.0)$ & $<0.0001$ \\
\hline \multicolumn{6}{|l|}{ Number of iv agents, n(\%) } \\
\hline 2 & $53 / 302(17.5)$ & $138 / 1,412(9.8)$ & $16 / 191(8.4)$ & $34 / 274(12.4)$ & $<0.0001$ \\
\hline$>3$ & 8/302 (2.6) & $51 / 1,412(3.6)$ & 2/191 (1.0) & 6/274 (2.2) & $<0.0001$ \\
\hline IV labetalol & $105 / 302(34.8)$ & $11 / 1,412(0.8)$ & 15/191 (7.9) & $54 / 274(19.7)$ & $<0.0001$ \\
\hline IV nicardipine & $1 / 302(0.3)$ & $14 / 1,412(1.0)$ & 109/191 (57.1) & $1 / 274(0.4)$ & $<0.0001$ \\
\hline IV urapidil & 6/302 (2.0) & 205/1,412 (14.5) & - & $4 / 274(1.5)$ & $<0.0001$ \\
\hline \multicolumn{6}{|l|}{ Oral agents used, $n(\%)$} \\
\hline 0 & $127 / 127(42.1)$ & $920 / 920(65.2)$ & $126 / 126(66.0)$ & $214 / 214(78.1)$ & $<0.0001$ \\
\hline 1 & 79/206 (26.2) & $345 / 1,265(24.4)$ & $32 / 158(16.8)$ & $34 / 248(12.4)$ & $<0.0001$ \\
\hline 2 & $54 / 260(17.9)$ & $120 / 1,385(8.5)$ & $30 / 188(15.7)$ & $14 / 262(5.1)$ & $<0.0001$ \\
\hline$>3$ & 42/302 (13.9) & 27/1,412 (1.9) & 3/191 (1.6) & $12 / 274(4.4)$ & $<0.0001$ \\
\hline Oral ACEI/ARB & 93/302 (30.8) & 199/1,412 (14.1) & 48/191 (25.1) & 27/274 (9.9) & $<0.0001$ \\
\hline Oral diuretic & 37/302 (12.3) & $56 / 1,412(4.0)$ & $11 / 191(5.8)$ & $14 / 274(5.1)$ & $<0.0001$ \\
\hline Oral beta blocker & 49/302 (16.2) & $87 / 1,412(6.2)$ & $8 / 191(4.2)$ & $14 / 274(5.1)$ & $<0.0001$ \\
\hline Oral calcium channel blocker & 69/302 (22.8) & 294/1,412 (20.8) & $30 / 191(15.7)$ & 29/274 (10.6) & 0.0002 \\
\hline Other oral sympathetic antagonist & 8/302 (2.6) & $5 / 1,412(0.4)$ & - & $2 / 274(0.7)$ & 0.0001 \\
\hline Other oral medication(s) & 70/302 (23.2) & 25/1,412 (1.8) & $5 / 191(2.6)$ & $15 / 274(5.5)$ & $<0.0001$ \\
\hline \multicolumn{6}{|l|}{ BP lowering treatment in days $2-7$} \\
\hline 1 & $56 / 275(18.7)$ & 298/1,218 (21.4) & 58/179 (30.9) & $78 / 241$ (28.6) & 0.0001 \\
\hline 2 & 19/294 (6.4) & $132 / 1,350(9.5)$ & 6/185 (3.2) & 19/260 (7.0) & 0.0001 \\
\hline$>3$ & $5 / 299(1.7)$ & 44/1,394 (3.2) & 3/188 (1.6) & 13/273 (4.8) & 0.0001 \\
\hline IV labetalol & $32 / 299(10.7)$ & $5 / 1,394(0.4)$ & $5 / 188(2.7)$ & 26/273 (9.5) & $<0.0001$ \\
\hline IV nicardipine & - & 7/1,394 (0.5) & 55/188 (29.3) & - & $<0.0001$ \\
\hline IV sodium nitroprusside & - & 88/1,394 (6.3) & $1 / 188(0.5)$ & 63/273 (23.1) & $<0.0001$ \\
\hline IV nitroglycerin & $58 / 299$ (19.4) & 64/1,394 (4.6) & 6/188 (3.2) & $14 / 273(5.1)$ & $<0.0001$ \\
\hline Other IV drug(s) & $35 / 299$ (11.7) & 252/1,394 (18.1) & 10/188 (5.3) & 40/273 (14.7) & $<0.0001$ \\
\hline \multicolumn{6}{|l|}{ Oral agents used, n(\%) } \\
\hline 0 & $62 / 299(20.7)$ & 716/1,394 (51.4) & $54 / 188(28.7)$ & 92/273 (33.7) & $<0.0001$ \\
\hline 1 & $71 / 299(23.7)$ & $350 / 1,394(25.1)$ & 62/188 (33.0) & $62 / 273(22.7)$ & $<0.0001$ \\
\hline 2 & 91/299 (30.4) & 202/1,394 (14.5) & $57 / 188(30.3)$ & 48/273 (17.6) & $<0.0001$ \\
\hline$>3$ & 75/299 (25.1) & 126/1,394 (9.0) & 15/188 (8.0) & 71/273 (26.0) & $<0.0001$ \\
\hline Oral ACEI/ARB & 145/299 (48.5) & 350/1,394 (25.1) & $94 / 188$ (50.0) & 135/273 (49.5) & $<0.0001$ \\
\hline Oral diuretic & 60/299 (20.1) & 158/1,394 (11.3) & 25/188 (13.3) & 71/273 (26.0) & $<0.0001$ \\
\hline Oral beta blocker & 85/299 (28.4) & 169/1,394 (12.1) & 22/188 (11.7) & 70/273 (25.6) & $<0.0001$ \\
\hline Oral calcium channel blocker & 102/299 (34.1) & 431/1,393 (30.9) & 70/188 (37.2) & 78/273 (28.6) & 0.1667 \\
\hline Other oral sympathetic antagonist & 9/299 (3.0) & 14/1,394 (1.0) & - & 15/273 (5.5) & $<0.0001$ \\
\hline
\end{tabular}


TABLE 2 | Continued

\begin{tabular}{|c|c|c|c|c|c|}
\hline & Western countries & China & Other asian & South America & $P$-value \\
\hline Other oral medication(s) & 104/299 (34.8) & 45/1,394 (3.2) & 12/188 (6.4) & $32 / 273(11.7)$ & $<0.0001$ \\
\hline \multicolumn{6}{|l|}{ BP lowering treatment at day 90} \\
\hline
\end{tabular}

Data are $n$ (\%). P-values are based on Chi-square test.

$B P$, blood pressure; IV, intravenous; $A C E I$, angiotensin converting enzyme inhibitors; $A R B$, angiotensin-receptor blockers.

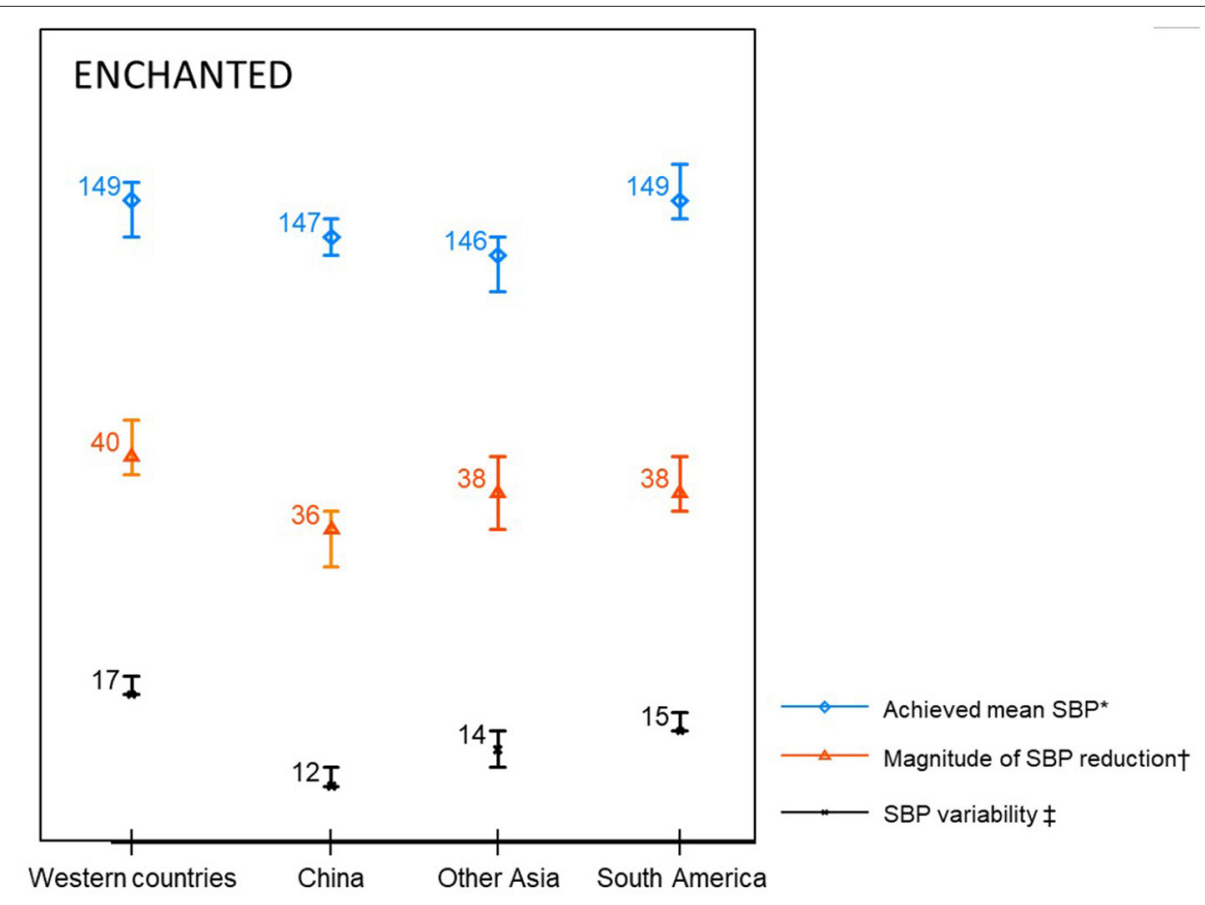

FIGURE 2 | Blood pressure parameters in the first $24 \mathrm{~h}$. SBP denotes systolic blood pressure. * Mean SBP in the first $24 \mathrm{~h} .{ }^{\dagger}$ SBP at randomisation minus minimum SBP within $24 \mathrm{~h}$. $\neq$ Standard deviation of SBP in the first $24 \mathrm{~h}$.

Patients from China, compared with other regions, were significantly less likely to receive BP-lowering treatment in both first $24 \mathrm{~h}$ and 2-7 days. In the first $24 \mathrm{~h}$, the most popular IV agents in China were urapidil (14.5\%) and sodium nitroprusside (12.4\%). More than a third of the patients from Western countries (36.4\%) were treated by IV nitroglycerin, followed by labetalol (34.8\%). More than a half of the patients in other Asian countries received nicardipine (57.1\%). In South America, most patients received sodium nitroprusside (39.4\%) and labetalol (19.7\%). For oral antihypertensive therapies, patients were more likely to take oral angiotensin converting enzyme (ACE) inhibitors/angiotensinreceptor blockers (ARBs) and calcium channel blockers in all four regions. The same pattern was also observed over 2-7 days (Figure 1 and Table 2).

Compared with other regions, patients from China had the least magnitude of SBP reduction and SBP variability throughout the first 7 days, and patients from Western countries had highest achieved mean SBP, magnitude of SBP reduction, and SBP variability over the first $24 \mathrm{~h}$ (Figure 2 and Table 3). Similar results were observed in both groups divided by randomization treatment (Supplementary Figure 1).

\section{DISCUSSION}

Our data adds evidence to regional differences in early BP management after AIS with patients from China being less likely to be treated with IV BP-lowering agents and less aggressively treated when compared with other regions. There are a number of possible explanations for this.

First, this may reflect ethnic and/or regional differences in hypertension management for both primary $(8,9)$ and secondary prevention (10) of stroke that have been reported from large-scale epidemiological studies. The PURE study (10) surveyed 7,519 participants with either a previous chronic heart disease event or stroke for use of cardiovascular eventpreventing drugs ( $\beta$-blockers, statins, antiplatelet therapy, or BPlowering drugs) for secondary prevention. Two thirds of the variation in antihypertensive drug use could be explained by 
TABLE 3 | BP control by region in ENCHANTED.

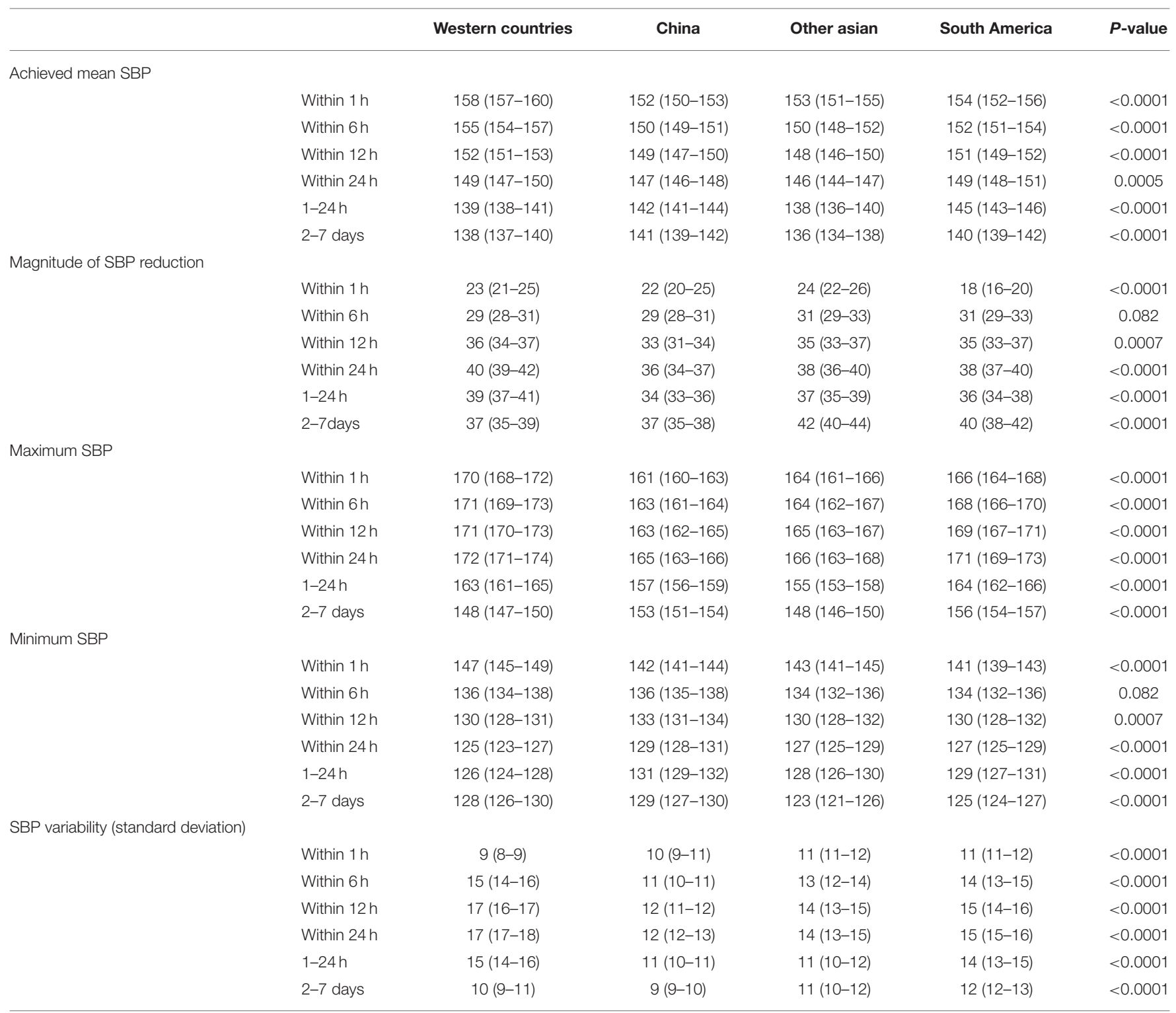

Data are mean (SD), or median (IQR). P-values are based on T-test, or Wilcoxon signed-rank test. $B P$, blood pressure, SBP, systolic blood pressure.

the economic status of the country, whereas only a third by individual factors (10). The Global Burden of Disease Study 2013 (8) and INTERSTROKE study (9) showed that the populationattributable risk of stroke due to hypertension was higher in Asian populations than in those of other ethnicities or regions. There are uncertainties in worldwide AIS clinical guidelines for blood pressure control, especially within $48 \mathrm{~h}$ after onset $(11,12)$.

Second, compared with other regions, there is a large number of patients with intracranial artery stenosis in China. This may influence Chinese clinicians' decision-making with concerns related to high risk of early neurological deficit with antihypertensive treatment in the AIS period. Accordingly, patients from China had a smaller magnitude of SBP reduction and SBP variability over the first $24 \mathrm{~h}$. This may relate to less use of BP-lowering treatment and less aggressive management with more than one type of IV agent. Overall, Chinese doctors are more conservative for BP-lowering treatment in acute stroke.

Third, Chinese patients were $\sim 10$ years younger than the Western patients and with fewer comorbidities, which may partly explain less need for BP-lowering therapy though these aspects were corrected in the multivariable analysis.

Finally, this may reflect regional differences in the use of IV BP-lowering agents. The lack of significant regional difference 
in oral BP-lowering drug use is likely to reflect drug classthiazide diuretics, ACE inhibitors, calcium antagonists, ARBs, and beta blockers-are equally effective with respect to the magnitude of BP reduction (approximately 10/5 mm Hg) when administered separately (Evidence Grade 1) (13). However, there is debate about the differential effect on reduction in stroke recurrence, which may be related to differences in BP variability measurements.

Key strengths of this study are the international recruitment of patients with a broad range of characteristics and use of a robust protocol with central randomization and objective outcome measures. Nonetheless, no information on the dose of BP-lowering agents was collected. This is a mainly descriptive study and prone to inadequate adjustment. In addition, intensive BP lowering was the main intervention in ENCHANTED, and this could affect antihypertensive treatment performance. The proportion of antihypertensive treatment may be overestimated. However, no specific drug was specified in the protocol, and the results can still show the regional differences in BP management.

In summary, regional variations appeared in early $\mathrm{BP}$ management, including receiving BP-lowering treatment, the intensity of the treatment, and BP-lowering agents used in AIS, which translated into the differences in hemodynamic parameters. Furthermore, regional differences need to be considered when conducting BP management trials and formulating blood pressure management guidelines.

\section{DATA AVAILABILITY STATEMENT}

De-identified participant data used in these analyses can be shared by formal request to the corresponding author.

\section{REFERENCES}

1. MacMahon S, Peto R, Cutler J, Collins R, Sorlie P, Neaton J, et al. Blood pressure, stroke, and coronary heart disease. Part 1, prolonged differences in blood pressure: Prospective observational studies corrected for the regression dilution bias. Lancet. (1990) 335:765-74. doi: 10.1016/0140-6736(90)90878-9

2. Oppenheimer S, Hachinski V. Complications of acute stroke. Lancet. (1992) 339:721-4. doi: 10.1016/0140-6736(92)90607-5

3. Britton M, Carlsson A, de Faire U. Blood pressure course in patients with acute stroke and matched controls. Stroke. (1986) 17:861-4. doi: 10.1161/01.STR.17.5.861

4. Chow CK, Teo KK, Rangarajan S, Islam S, Gupta R, Avezum A, et al. Prevalence, awareness, treatment, and control of hypertension in rural and urban communities in high-, middle-, and low-income countries. JAMA. (2013) 310:959-68. doi: 10.1001/jama.2013.184182

5. Rahimi K, Emdin CA, MacMahon S. The epidemiology of blood pressure and its worldwide management. Circ Res. (2015) 116:925-36. doi: 10.1161/CIRCRESAHA.116.304723

6. Huang Y, Sharma VK, Robinson T, Lindley RI, Chen X, Kim JS, et al. Rationale, design, and progress of the enhanced control of hypertension and thrombolysis stroke study (enchanted) trial: an international multicenter $2 \mathrm{x}$ 2 quasi-factorial randomized controlled trial of low- vs. Standard-dose rt-pa and early intensive vs. Guideline-recommended blood pressure lowering in patients with acute ischaemic stroke eligible for thrombolysis treatment. Int J Stroke. (2015) 10:778-88. doi: 10.1111/ijs.12486
Requests to access these datasets should be directed to Craig Anderson, canderson@georgeinstitute.org.au.

\section{ETHICS STATEMENT}

The studies involving human participants were reviewed and approved by Australia Royal Prince Alfred Hospital HREC. The patients/participants provided their written informed consent to participate in this study.

\section{AUTHOR CONTRIBUTIONS}

LS, CA, and XW contributed to study design, organization, statistical review, and critique of the report, and XW undertook analyses. CC drafted the manuscript. All authors contributed to the concept and rationale for the study, made critical revisions, approved the final article, and take responsibility for its content and integrity.

\section{FUNDING}

Funding was primarily from the National Health and Medical Research Council (NHMRC) of Australia. Additional funding was from the Stroke Association of the United Kingdom, the National Council for Scientific and Technological Development of Brazil, and the Ministry for Health, Welfare and Family Affairs of the Republic of Korea (HI14C1985).

\section{SUPPLEMENTARY MATERIAL}

The Supplementary Material for this article can be found online at: https://www.frontiersin.org/articles/10.3389/fneur. 2021.687862/full\#supplementary-material

7. Anderson CS, Huang Y, Lindley RI, Chen X, Arima H, Chen $\mathrm{G}$, et al. Intensive blood pressure reduction with intravenous thrombolysis therapy for acute ischaemic stroke (enchanted): an international, randomised, open-label, blinded-endpoint, phase 3 trial. Lancet. (2019) 393:877-88. doi: 10.1016/S0140-6736(19) 30038-8

8. Feigin VL, Roth GA, Naghavi M, Parmar P, Krishnamurthi R, Chugh S, et al. Global burden of stroke and risk factors in 188 countries, during 1990-2013: a systematic analysis for the global burden of disease study (2013). Lancet Neurol. (2016) 15:913-24. doi: 10.1016/S1474-4422(16)3 0073-4

9. O’Donnell MJ, Chin SL, Rangarajan S, Xavier D, Liu L, Zhang H, et al. Global and regional effects of potentially modifiable risk factors associated with acute stroke in 32 countries (interstroke): a casecontrol study. Lancet. (2016) 388:761-75. doi: 10.1016/S0140-6736(16) 30506-2

10. Yusuf S, Islam S, Chow CK, Rangarajan S, Dagenais G, Diaz $\mathrm{R}$, et al. Use of secondary prevention drugs for cardiovascular disease in the community in high-income, middle-income, and lowincome countries (the pure study): a prospective epidemiological survey. Lancet. (2011) 378:1231-43. doi: 10.1016/S0140-6736(11) 61215-4

11. Society CNC. Chinese guidelines for diagnosis and treatment of acute ischemic stroke 2018. Chin J Neurol. (2018) 51:666-82. doi: 10.3760/cma.j.issn.1006-7876.2018.09.004 
12. Warner JJ, Harrington RA, Sacco RL, Elkind MSV. Guidelines for the early management of patients with acute ischemic stroke: 2019 update to the 2018 guidelines for the early management of acute ischemic stroke. Stroke. (2019) 50:3331-2. doi: 10.1161/STROKEAHA.119.027708

13. Swedish Council on Health Technology A. Sbu Systematic Review Summaries. Moderately Elevated Blood Pressure: A Systematic Review. Stockholm: Swedish Council on Health Technology Assessment (SBU).

Author Disclaimer: The views expressed in this article are those of the author(s) and not necessarily those of the NIHR or Department of Health and Social Care.

Conflict of Interest: LS, HA, and CA report speaking fees from Takeda China. TR is a National Institute for Health Research (NIHR) Senior Investigator; JC reports grants from Servier and NHMRC. CA holds a Senior Investigator Fellowship and grants from NHMRC and Takeda China. XW is supported by National Heart Foundation postdoctoral fellowship (102117); New South Wales Health commission investigator development grant; and NHMRC investigator grant (APP1195237).
The remaining authors declare that the research was conducted in the absence of any commercial or financial relationships that could be construed as a potential conflict of interest.

Publisher's Note: All claims expressed in this article are solely those of the authors and do not necessarily represent those of their affiliated organizations, or those of the publisher, the editors and the reviewers. Any product that may be evaluated in this article, or claim that may be made by its manufacturer, is not guaranteed or endorsed by the publisher.

Copyright (c) 2021 Chen, Song, Yang, Lindley, Robinson, Arima, Chalmers, Anderson and Wang. This is an open-access article distributed under the terms of the Creative Commons Attribution License (CC BY). The use, distribution or reproduction in other forums is permitted, provided the original author(s) and the copyright owner(s) are credited and that the original publication in this journal is cited, in accordance with accepted academic practice. No use, distribution or reproduction is permitted which does not comply with these terms. 